\title{
A family of small cyclic amphipathic peptides (SCAmpPs) genes in citrus
}

\author{
William R Belknap ${ }^{1 *}$, Kent F McCue ${ }^{1}$, Leslie A Harden, William H Vensel', Michael G Bausher ${ }^{2}$ and Ed Stover ${ }^{2}$
}

\begin{abstract}
Background: Citrus represents a crop of global importance both in economic impact and significance to nutrition. Citrus production worldwide is threatened by the disease Huanglongbing (HLB), caused by the phloem-limited pathogen Candidatus Liberibacter spp.. As a source of stable HLB-resistance has yet to be identified, there is considerable interest in characterization of novel disease-associated citrus genes.

Results: A gene family of Small Cyclic Amphipathic Peptides (SCAmpPs) in citrus is described. The citrus genomes contain 100-150 SCAmpPs genes, approximately 50 of which are represented in the citrus EST database. These genes encode small $\sim 50$ residue precursor proteins that are post-translationally processed, releasing 5-10 residue cyclic peptides. The structures of the SCAmpPs genes are highly conserved, with the small coding domains interrupted by a single intron and relatively extended untranslated regions. Some family members are very highly transcribed in specific citrus tissues, as determined by representation in tissue-specific cDNA libraries. Comparison of the ESTs of related SCAmpPs revealed an unexpected evolutionary profile, consistent with targeted mutagenesis of the predicted cyclic peptide domain. The SCAmpPs genes are displayed in clusters on the citrus chromosomes, with apparent association with receptor leucine-rich repeat protein arrays. This study focused on three SCAmpPs family members with high constitutive expression in citrus phloem. Unexpectedly high sequence conservation was observed in the promoter region of two phloem-expressed SCAmpPs that encode very distinct predicted cyclic products. The processed cyclic product of one of these phloem SCAmpPs was characterized by LC-MS-MS analysis of phloem tissue, revealing properties consistent with a $\mathrm{K}^{+}$ionophore.
\end{abstract}

Conclusions: The SCAmpPs amino acid composition, protein structure, expression patterns, evolutionary profile and chromosomal distribution are consistent with designation as ribosomally synthesized defense-related peptides.

Keywords: Citrus sinensis, Citrus clementina, Poncirus trifoliata, Ribosomally synthesized

\section{Background}

Plants produce a broad array of peptides, short proteins of approximately 50 residues or less, largely involved in defense processes [1]. With the exception of phytochelatins [2], these plant peptides are ribosomally synthesized either as mature products or precursors that are posttranslationally modified [3]. The plant peptides vary in size, composition, and mode of action, and many have been associated with defense against pathogens and predators.

These defense-related peptides accumulate either constitutively or are induced by injury/infection [4]. The plant defensins, for example, are encoded by a multigene family with small (45-54 residue) cysteine-rich protein products.

\footnotetext{
* Correspondence: william.belknap@ars.usda.gov

'USDA-ARS, Western Regional Research Center, Albany, CA, USA

Full list of author information is available at the end of the article
}

While defensins can constitutively accumulate in plant tissues, specific family members are induced by a variety of biotic and abiotic stresses [4]. Defensin gene products have been shown to have anti-microbial activities, and have been successfully employed as transgene products in number of plants, including citrus [5].

In a study designed to identify citrus genes transcriptionally up-regulated by insect herbivory, a short transcript encoding an unknown 50-residue protein was identified [6]. Designated CsV03-3, in citrus leaves this transcript was shown to accumulate in response to application of methyl jasmonate, salicylic acid, abscisic acid and abiotic stress [7]. Three additional transcripts with $>90 \%$ identity to CsV03-3 were identified in the EST database, indicating that it was a member of a gene family. The small proteins encoded by the transcripts were shown to bind dsDNA, 
and the family was designated as a new class of defenserelated nucleic acid binding proteins [7]. However, unrelated investigations of citrus peptides with potential pharmaceutical applications [8,9] identified small cyclic citrus peptides with precursors closely related to the $\mathrm{CsV} 03$ family, suggesting CsV03 membership in a more diverse gene family encoding cyclic peptides [10].

Here we describe the structure, expression, evolutionary profile and genomic distribution of members of a diverse gene family of CsV03-related SCAmpPs. In addition, we show that one of the members, highly expressed in phloem tissue, encodes a cyclic peptide with the potential to act as a $\mathrm{K}^{+}$carrier ionophore.

\section{Results}

\section{SCAmpPs Peptides}

While the four previously described CsV03 transcripts were identified as encoding nucleic acid binding proteins [7], these proteins show considerable similarity to three cyclic-peptide precursors characterized in unrelated studies (Figure 1a) [8-10]. The two cyclic peptides from C. aurantium as well as the one from $C$. natsudaidai are in all likelihood derived from precursor proteins closely related to the four CsV03 proteins (Figure 1c) [10]. This implies that the CsV03 genes belong to a larger, and more diverse, family than previously indicated [7]. It has recently been suggested that small peptides cyclized $\mathrm{N}$-to-C and lacking disulfides be referred to as orbitides [3].

In order to more fully characterize this gene family, a consensus sequence (Figure 2) was used to probe the $C$. sinensis [11] and C. clementina [12] genome assemblies. A total of 102 loci with similarity to the consensus sequence were identified on the C. clementina genome (Additional file 1), and of these 36 were associated with ESTs ( $\geq 99 \%$ identity) indicating that they represent expressed genes. A total of 150 similar loci were identified on the C. sinensis draft genome, 46 of which are associated with ESTs. The expressed genes had the structure indicated on Figure 1b, with two exons separated by an approximately $100 \mathrm{bp}$ intron. While the coding sequences of these genes (approximately150 bp) are short, the transcripts contain relatively long 5 ' and 3 ' UTRs resulting in transcripts of approximately 450 bp (Figure 1b). Thirtythree of the expressed genes in C. clementina have at least

\begin{tabular}{|c|c|c|c|c|}
\hline \multirow{2}{*}{ a } & \multicolumn{4}{|c|}{$\begin{array}{l}\text { Cyclic } \\
\text { Peptide }\end{array}$} \\
\hline & $\begin{array}{l}\text { SCAmpPs1 } \text { CsV03-1 } \\
\text { SCAmpPs2 CsV03-2 } \\
\text { SCAmpPs CsV03-3 } \\
\text { SCAmpPs4 CsV03-4 } \\
\text { Citrus aurantium-1 } \\
\text { Citrus aurantium-2 } \\
\text { Citrus natsudaidai }\end{array}$ & $\begin{array}{l}\text { MKIMETTCAGNDDCLE } \\
\text { MKNMETTCAGNDDWLE } \\
\text { MKNMETTSAGNDDWLE } \\
\text { MKNMETTSAGNADWLE } \\
\text { METTCAGNN-WSE } \\
\text { MKTLAGAGMSDPSE } \\
\text { MKTLPGAGMSDPSE }\end{array}$ & $\begin{array}{l}\text { GRPWNLA } \\
\text { GAPWLIAA } \\
\text { GYVAA } \\
\text { GVPWAIAA } \\
\text { GLLLPPFG } \\
\text { GLVLPS } \\
\text { GYLLPPS }\end{array}$ & $\begin{array}{l}\text { SIVDDNVANDVNLDLLAVPQYGRNTDQTG * } \\
\text { SIVDDNIANDVNLDLLTVPQYGRNIDQTG * } \\
\text { SIVDDNIANDVNLDLLTVPQYGRNIDQTG * } \\
\text { SIVDDNIANDVNLDLLTVPQYGRNIDQTG * } \\
\text { SIADDDVMND - NLDFLNVPQYGRNPDYMG * } \\
\text { SIADDDVGND - NLDLIVIPQYGRNPDYYG * } \\
\text { SIADDDVGND - NLDLIVIPQYGRNPDYYG * }\end{array}$ \\
\hline & \multicolumn{3}{|c|}{$\begin{array}{l}\text { Cyclic } \\
\text { Peptide }\end{array}$} & \multirow[b]{2}{*}{ Exon2 } \\
\hline & Exon1 & Intron & & \\
\hline & 100 & 200 & 300 & 500 \\
\hline & $\begin{array}{l}\text { C. Clem. Chr5 } 7.138 \\
\text { Citrus aurantium-1 }\end{array}$ & $\begin{array}{l}\text { METTCAGNNWSE GFL } \\
\text { METTCAGNNWSE GLL }\end{array}$ & \begin{tabular}{l|l} 
LIPAA- & SIA \\
LLPPFG & SIA \\
\end{tabular} & $\begin{array}{l}\text { S IADDDVMNDNLDLLNI PQYGRNPDYMG * } \\
\text { S IADDDVMNDNLDFLNVPQYGRNPDYMG * }\end{array}$ \\
\hline & $\begin{array}{l}\text { C. Clem. Chr5 } 1.003 \\
\text { citrus natsudaidai } \\
\text { Citrus aurantium-2 }\end{array}$ & \begin{tabular}{l} 
MKTLPGAGMSDPSE \\
MKY \\
MKTLPGAGMSDPSE \\
MKTLAGAGMSDPSE \\
\cline { 2 - 3 }
\end{tabular} & \begin{tabular}{l|l} 
YLLPPS & SIA \\
YLLPPS & SIA \\
SVLP-S & SIA \\
\end{tabular} & $\begin{array}{l}\text { ADDDVGNDNLDLIVI PQYGRNPDYYG* } \\
\text { ADDDVGNDNLDLIVI PQYGRNPDYYG * } \\
\text { ADDDVGNDNLDLIVI PQYGRNPDYYG * }\end{array}$ \\
\hline \multicolumn{5}{|c|}{$\begin{array}{l}\text { Figure } 1 \text { Alignment of SCAmpPs precursor proteins and structure of SCAmpPs genes. (a) Alignment of the precursor deduced amino acid } \\
\text { sequences SCAmpPs-1 (NCBI accession EF175924), SCAmpPs-2 (EF175926), SCAmpPs-3 (EF175925), SCAmpPs-4 (EF175927), Citrus aurantium-1 } \\
\text { (EY850721), Citrus aurantium-2 (EY848546) and Citrus natsudaidai (BB999724). Boxes indicate predicted cyclic peptide product domains. } \\
\text { (b) Representation of a model citrus SCAmpPs gene. Locations of precursor codons are indicated in dark blue, cyclic peptide product } \\
\text { codons in light blue. Exon and intron positions indicated by brown and white boxes. (c) Deduced amino acid sequences of C. clementina } \\
\text { SCAmpPs located on chromosome } 5 \text { at positions } 7.138 \text { (NCBI accession FC928056) and 1.003 Mb (FC872825). }\end{array}$} \\
\hline
\end{tabular}




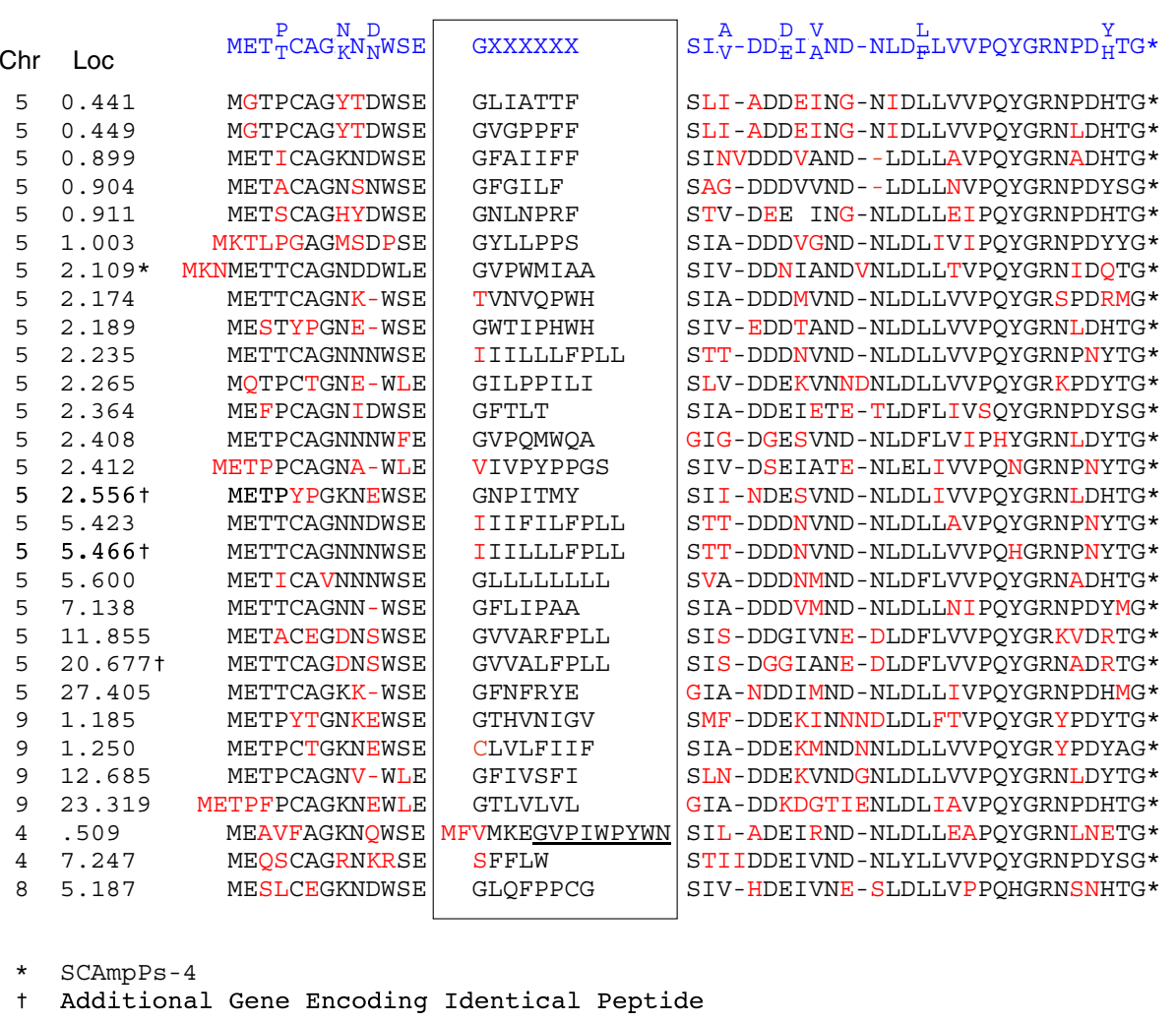

Figure 2 Alignment of SCAmpPs precursor proteins. Deduced SCAmpPs precursor protein sequences from citrus for gene products found in both the Citrus sinensis and Citrus clementina genomes as well as the citrus EST database. The predicted cyclic peptide product sequences are indicated, as well as the chromosome number and position (Mb) on the encoding gene Citrus clementina genome. The SCAmpPs precursor consensus sequence is indicated in blue.

99\% identity to the $C$. sinensis orthologs. The deduced amino acid sequences of the encoded precursor proteins, verified in two independent assemblies and the EST database, are aligned in Figure 2.

The alignments shown in Figure 1c support identification of the proteins in Figure 2 as precursors processed to the small predicted cyclic final products indicated. All three of the previously verified citrus precursors $[8,9]$ align with specific expressed $C$. clementina deduced proteins. As a majority of the deduced cyclic products contain a combination of both non-polar/hydrophobic, and polar/charged residues, members of this gene family are referred to as Small Cyclic Amphipathic Peptides, or SCAmpPs. The previously described cyclic proteins from Citrus aurantium and Citrus natsudaidai are clearly members of this set (Figure 1c).

The SCAmpPs precursor product from $0.509 \mathrm{Mb}$ on C. clementina chromosome 4 (Figure 2) has been entered to align the -WSE- amino-terminal processing site proposed for the other precursors. However, the structural features of the deduced precursor suggests that processing of this peptide results in the underlined GVPIWPYWN predicted cyclic peptide.
The processing of the Caryophyllaceae orbitide precursors in developing seeds to final, cyclic, products has been shown to occur in two steps $[10,13]$. Initially, the precursors are cleaved at the N-terminal end of the cyclic product by an oligopeptidase, followed by secondary cleavage at the cyclic peptide $\mathrm{C}$-terminus and formation of the final product via a specific cyclase [13]. While there is no sequence similarity between the citrus and Caryophyllaceae precursors, their structural similarity [10] suggests a similar biosynthetic pathway.

The amino acid content of the SCAmpPs predicted cyclic peptides are enriched in non-polar amino acids (tryptophan, phenylalanine, proline, leucine, isoleucine) relative to expected frequencies [14], and while there is an overall decrease in charged residues, the ratio of basic to acidic amino acids is high in the SCAmpPs peptides (Additional file 2). This composition is consistent with peptide attachment and insertion into biological membranes [15].

\section{SCAmpPs transcription}

Based on representation in the available citrus EST database [16], the individual SCAmpPs gene family members 
can have strikingly different transcriptional profiles (Additional file 3). For example representation of individual SCAmpPs in cDNA libraries from C. sinensis phloem (NCBI LIBEST_017673, Bausher, M., Shatters, R., Dang, P., Hunter, W. and Niedz, R., unpublished, 2005) and C. clementina ovary abscission zone C (NCBI LIBEST_019157 [17]) can be compared. SCAmpPs-2, SCAmpPs-3 and SCAmpPs- 4 are highly represented in the phloem and under-represented in the ovary abscission zone, while the reverse is true of SCAmpPs-5 and SCAmpPs-6 (Additional file 3).

A sequence comparison of ESTs encoding SCAmpPs-3 and SCAmpPs-4 is shown in Figure 3. These ESTs are identical prior to the intron, and diverge thereafter. While these mRNAs share $97 \%$ identity outside the predicted cyclic peptide domains, the cyclic peptide coding sequences more divergent and result in five residue SCAmpPs-3 (GYVAA) and eight residue SCAmpPs-4 (GVPWAIAA) cyclic peptide products that share only three common residues. A hypervariable domain is clearly apparent in the alignment as the domain encoding the final cyclic product. These two mRNAs share $100 \%$ identity in the domain $5^{\prime}$ to the intron processing locus with less conservation 3 ' to the cyclic product.

The pattern of sequence divergence, specifically targeting the predicted cyclic peptide-coding domain, is observed in other SCAmpPs ESTs with similar expression profiles (based on representation in the database). For example, an alignment of SCAmpPs-5 and SCAmpPs-6 ESTs is shown in Figure 4a. While these ESTs share 97\% identity outside the cyclic peptide domain (95\% overall identity), the cyclic peptide domains share only $43 \%$ identity resulting in cyclic peptides (SCAmpPs-5 GGAPPWF, SCAmpPs-6 GLIATTF) that share only two common residues (Figure 4a). This pattern is also observed in comparison of the SCAmpPs-5 EST with an orthologous EST from $P$. trifoliata, an evolutionary divergence of approximately 4 million years [18]. These transcripts share 95\% identity outside the cyclic peptide domain, while the localized changes in the cyclic peptide domain result in 7 and 8 residue peptides that share a single common residue (Figure $4 \mathrm{~b}$ ).

The pattern of hypervariability within the predicted cyclic peptide-coding region is a common feature within paralogous/orthologous collections of SCAmpPs-encoding ESTs (Additional file 4). This type of hypervariability is common in gene families that mediate interactions between organisms, the conotoxins (snail toxins) providing perhaps the best example [19]. The accelerated mutation rates associated with the cyclic peptide domains could reflect either a strong positive selection or specific hypermutation. In the conotoxin case, a targeted mutagenic process producing short hypervariable stretches within the mature toxin domain has been proposed [20]. Alternative mechanisms for targeted changes within the conotoxin coding region based on formation of highly stable secondary structures within conserved regions [21] do not appear applicable in the SCAmpPs genes as they lack obvious potential for such secondary structures in the conserved regions.

\section{Genomic structure of the citrus phloem SCAmpPs-3 and SCAmpPs-4 genes}

While coding for distinct cyclic peptide products, the first exons of SCAmpPs- 3 and SCAmpPs- 4 mRNAs are identical (Figure 3). The C. sinensis Chr3 assembly [11] contains single SCAmpPs-3 (3.555 Mb, contains two tandem transcribed regions separated by $200 \mathrm{bp}$ ) and

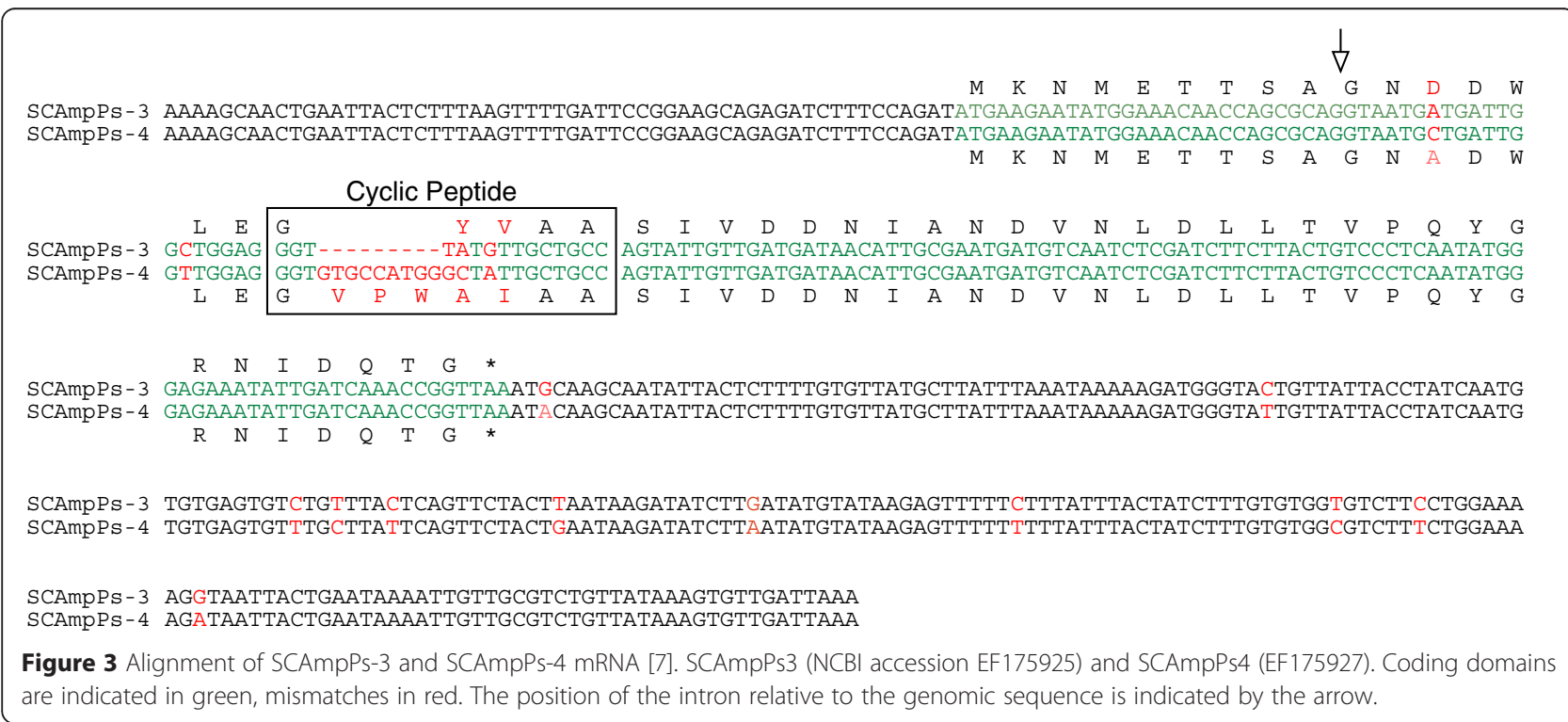




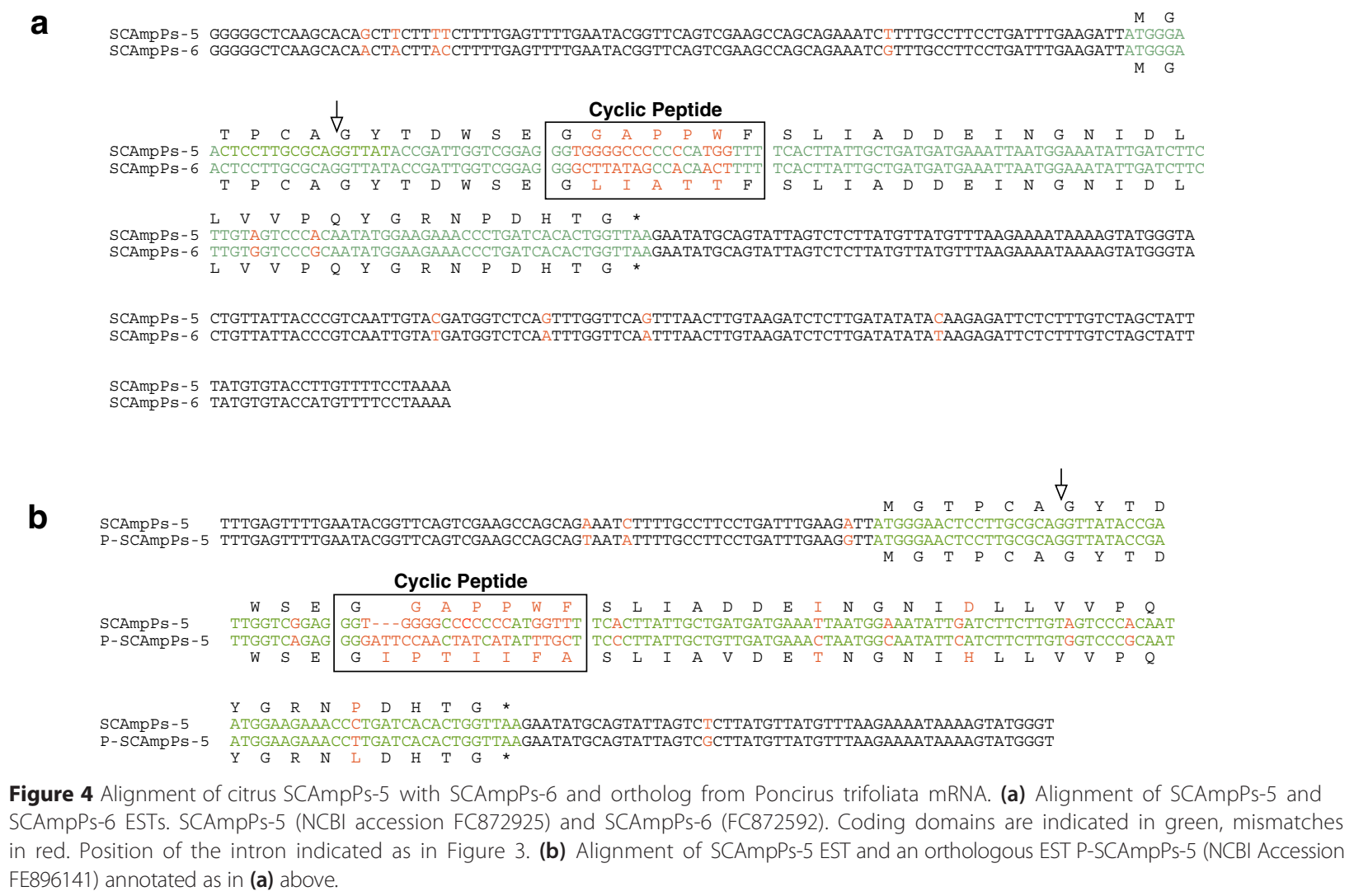

b

Figure 4 Alignment of citrus SCAmpPs-5 with SCAmpPs-6 and ortholog from Poncirus trifoliata mRNA. (a) Alignment of SCAmpPs-5 and SCAmpPs-6 ESTs. SCAmpPs-5 (NCBI accession FC872925) and SCAmpPs-6 (FC872592). Coding domains are indicated in green, mismatches in red. Position of the intron indicated as in Figure 3. (b) Alignment of SCAmpPs-5 EST and an orthologous EST P-SCAmpPs-5 (NCBI Accession FE896141) annotated as in (a) above.

SCAmpPs-4 (3.635 Mb) loci. The SCAmpPs-3 and SCAmpPs-4 genes in this assembly have similar $5^{\prime}$ domains, with a $1.9 \mathrm{~kb}$ relative deletion $200 \mathrm{bp} 5^{\prime}$ to the SCAmpPs-3 transcription start. The C. clementina Chr5 assembly [12] does not contain a SCAmpPs-3 gene, but has a SCAmpPs-4 gene at $2.109 \mathrm{Mb}$ with a $5^{\prime}$ domain similar to the C. sinensis SCAmpPs-4 gene. The C. clementina Chr5 assembly also contains a SCAmpPs-4 -related pseudogene at $2.118 \mathrm{Mb}$. To evaluate the extent of conservation between these two genes, PCR primers were designed corresponding to $5^{\prime}$ and $3^{\prime}$ sequences conserved on both SCAmpPs-4 genes as well as C. sinensis SCAmpPs-3. These primers were used to amplify the genes from $C$. clementina genomic DNA.

The primers were expected to amplify products with $3.1 \mathrm{~kb}$ of $5^{\prime}$ and $250 \mathrm{bp}$ of $3^{\prime}$ sequence in relation to the SCAmpPs-4 gene on the $C$. sinensis Chr3 assembly. The sequences of two SCAmps-3 and three SCAmpPs-4 genes amplified from $C$. clementina were determined, as well as a SCAmpPs-3 gene amplified from the rootstock Carrizo. The source of the $5^{\prime}$ identity of the SCAmpPs-3 and SCAmpPs-4 ESTs (Figure 3) is shown in Figure 5, in which the sequences of a SCAmpPs-3 and SCAmpPs-4 genomic PCR products are compared. The two PCR products (gSCAmpPs-3-1 and gSCAmpPs-4-2) with a 5' promoter element of 3109 bp share identical sequence from a position $6 \mathrm{bp} 3^{\prime}$ to the intron (Figure 3) to a position $-2.5 \mathrm{~kb}$ relative to the transcription start. These two genes, which produce very different predicted cyclic products (Figure 1), therefore share the same $2.5 \mathrm{~kb}$ proximal promoter sequence, as well as first exon and intron sequences. The two additional SCAmpPs-4 PCR products had a $5^{\prime}$ sequence of 3128 bp due to a short variable region (46 or $65 \mathrm{bp)} 130 \mathrm{bp} 5^{\prime}$ to the transcription start (Figure 5, Additional file 5). Upstream of this variable domain the promoter sequences for the SCAmpPs-3 and SCAmpPs-4 genes were essentially identical (a single SCAmpPs PCR product- clone5- had a 1 bp deletion in this domain). The level of identity in the $5^{\prime}$ domains is consistent with the similar representation of SCAmpPs-3 and SCAmpPs-4 ESTs in the citrus phloem cDNA library (Additional file 3).

The level of sequence identity among the SCAmpPs-3 and SCAmpPs-4 genes will clearly prevent accurate assembly of individual members from short sequencing reads. The $C$. sinensis genome assembly [11] contains the intact gSCAmpPs-4-2 gene (Chr3 3.635) as well as two linked partial gSCAmpPs-3-1 genes (Chr3 $3.555 \mathrm{Mb}$ ). The $C$. clementina assembly contains two SCAmpPs-4related pseudogenes at 2.109 $\mathrm{Mb}$ (Additional file 6) and $2.118 \mathrm{Mb}$ on Chr5, both interrupted by repetitive DNA sequences. 


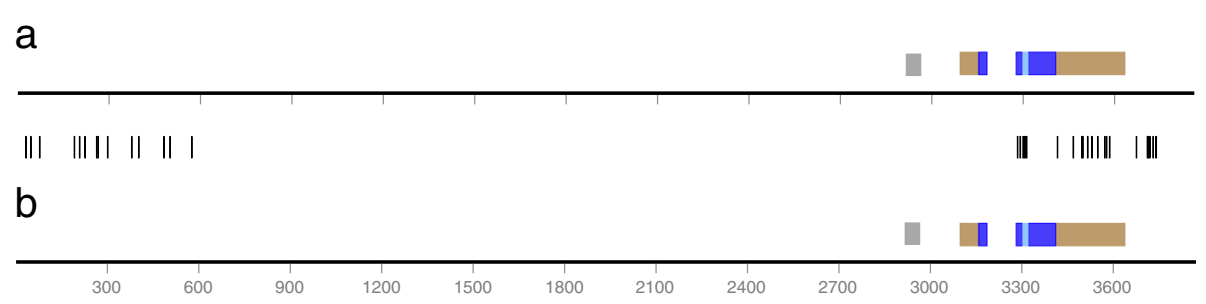

Figure 5 PCR Amplification of SCAmpPs-3 and SCAmpPs-4 genes. (a) gSCAmpPs-3-1, (b) gSCAmpPs-4-2. Exon, coding and cyclic peptide regions are indicated as in Figure 1. The grey boxes indicate the variable domains (Additional file 5). The black lines between the two sequences indicate the positions of single base pair differences.

\section{SCAmpPs genomic architecture}

As shown in Figure 6a, 66 of the 102 SCAmpPs-related C. clementina genomic loci are located in the first $20.86 \mathrm{Mb}$ of chromosome 5 [12]. Within this region, the distribution of SCAmpPs is non-random, most are present as members of localized SCAmpPs gene clusters [22]. This genomic region is also rich in arrays of Nucleotide Binding-Leucine Rich Repeat Protein (NBLRRP) disease resistance gene arrays [23], several of which are proximal to the SCAmpPs gene assemblies. The clearest example of this is shown in Figure 6b. The region 2.50-2.89 $\mathrm{Mb}$ contains 13 tandemly repeated RPP13-like [24] NB-LRRP genes and 13 SCAmpPs genes. The duplications giving rise to this array resulted in duplication of a proximal SCAmpPs gene. Figure 6c showed the chromosome 5 region $5.27-5.96 \mathrm{Mb}$ in which a SCAmpPs array is also flanked by duplicated NB-LRRP genes.

\section{Identification of SCAmpPs-4 Cyclic product by LC-MS}

The SCAmpPs cyclic peptide products in Figure 2 are assigned based on precursor protein similarity to previously identified cyclic products (Figure 1) [8,9]. Mass spectral analysis of C. clementina phloem proteins was employed to verify this assignment. The molecular ion $\left(\mathrm{MH}^{+}\right)$of the cyclic SCAmpPs-4 peptide GVPWAIAA, has a calculated monoisotopic mass of 766.4252. The LC-MS profile of the phloem extract was compared to synthetic cyclic SCAmpPs-4 product in the expected molecular mass range $(766.32-766.52 \mathrm{~m} / \mathrm{z}$ ) (Figure 7). The equivalence of the synthetic SCAmpPs-4 and the cyclic product from the phloem was established by consistent HPLC retention times and MS-MS HCD fragmentation of $\mathrm{m} / \mathrm{z} 766.42$ (Additional file 7).

The MS characterization of the SCAmpPs- 4 chromatographic peak for both the synthetic peptide and the $C$. clementina phloem extract are shown in Additional file 8 . The spectra averaged across the $\mathrm{LC}$ peak at the expected protonated cyclic peptide mass $(766.42 \mathrm{~m} / \mathrm{z})$ also contains a separate, higher mass peak. The higher mass peak in the synthetic peptide sample $(788.40 \mathrm{~m} / \mathrm{z})$ is consistent with the calculated molecular mass of the sodium adduct of the cyclic peptide (Additional file 8a). In contrast, the major peak in the phloem sample, measured as $804.38 \mathrm{~m} / \mathrm{z}$ (Additional file $8 \mathrm{~b}$ ) is consistent with the calculated molecular mass of the potassium adduct of the cyclic peptide. Considering the LC conditions were the same in both cases, the relative intensity of the potassium adduct of the phloem sample compared to the synthetic sample indicates the source of the potassium, and therefore adduct, is the phloem. The relative difference in intensity is thought to be reflective of solution-phase binding [25]. MS-MS fragmentation of mass $804.38 \mathrm{~m} / \mathrm{z}$ revealed minimal fragmentation at the HCD relative collision energy of 35 (data not shown) in contrast to significantly greater fragmentation observed for the non-adducted peptide, giving further evidence of stability of the molecule when complexed with potassium (Additional file 7).

To date, no biological role has been assigned to any of the orbitides [3]. Both the structure and cation binding properties of the SCAmpPs-4 (Additional file 7) open the possibility that this cyclic peptide represents a $\mathrm{K}^{+}$ionophore [26]. This interpretation is also consistent with the fragmentation pattern of the $\mathrm{K}^{+}$adduct, resulting from the conformational changes expected in the $\mathrm{K}^{+}$complexed relative to the uncomplexed form of the cyclic peptide [27]. While alkali metal ion adducts are commonly observed in electrospray, the potassium adduct is observed only in the plant-derived sample (Additional file 8).

\section{Discussion}

The biosynthesis of cyclic peptides occurs either through non-ribosomal pathways [28] or processing of ribosomally synthesized precursors [10]. Similar to Citrus, the Caryophyllaceae family members produce an array of 5-12 residue ribosomally synthesized small cyclic peptides derived from short precursor proteins [10]. Characterization of cyclic peptide biosynthesis in Saponaria vaccaria 


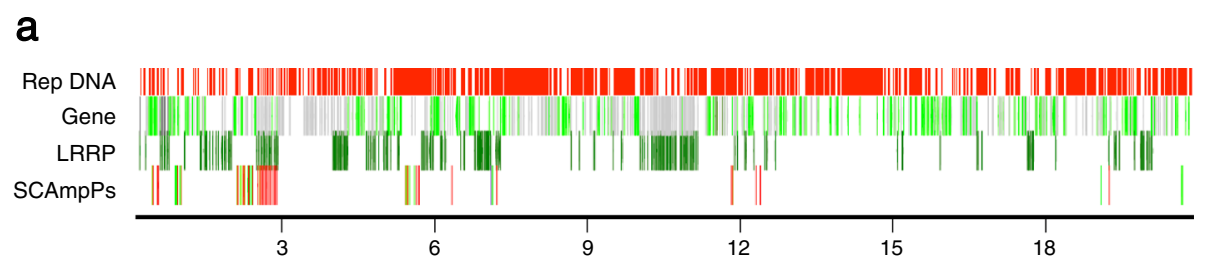

b

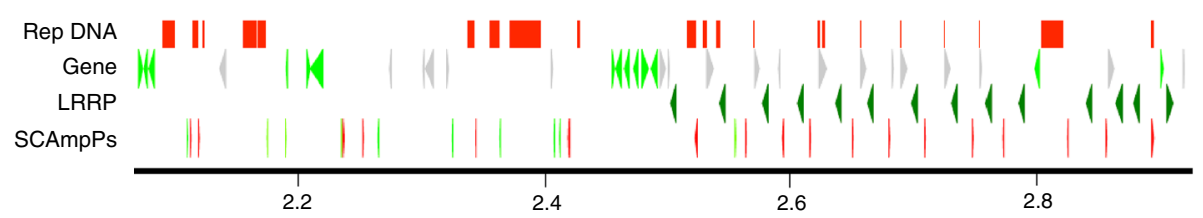

C

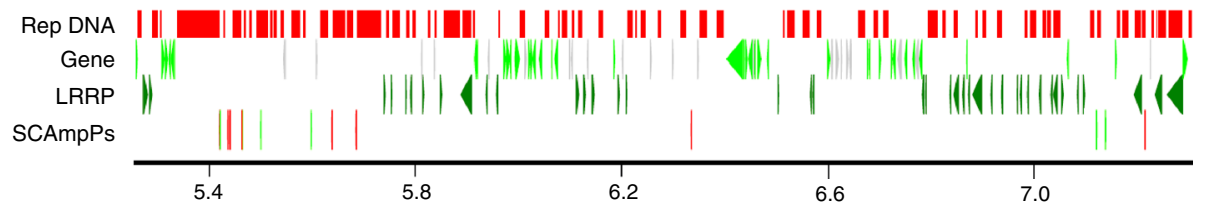

Figure 6 Distribution of SCAmpPs, Leucine-Rich Repeat Proteins, genes and repetitive DNA on Citrus clementina chromosome 5. (a) Positions 1-20.86 Mb of Chr5. SCAmpPs genes represented in the EST database are indicated in green, those not so represented in red. LRRP genes and pseudogenes are indicated in green. Other genes represented in the EST database are indicated in green, those not so represented are in grey. Ruler indicates position in Mb. (b) C. clementina Chr5 positions 2.07-2.92 Mb. (c) C. clementina Chr5 positions 5.25-7.30.

revealed the two-step processing of a precursor, involving an oligopeptidase cleavage of an amino terminal domain followed by an internal cyclization step resulting in removal of carboxy terminal residues [13]. With the exception of a conserved glycine residue at the amino terminal end of the cyclic peptide domain, the precursors for the Saponaria vaccaria peptides and citrus SCAmpPs share no sequence similarity. However, the precursor structures suggest a similar biosynthetic pathway in citrus [10].

Previously, SCAmpPs gene products have been proposed to be defense-related based on transcriptional profiles [7]. This interpretation is supported both by the structure of the final peptide product [29] and the chromosomal distribution of the SCAmpPs genes (Figure 6) [22]. The evolutionary profile of the SCAmpPs genes, in particular hypervariability of the predicted cyclic peptide domain, is also consistent with a defensive role [19,20,30].

The SCAmpPs composition, structure, expression, evolutionary profile and chromosomal distribution are consistent with designation as ribosomally synthesized antimicrobial peptides (AMPs) [1,31]. SCAmpPs predicted cyclic products are generally amphiphilic in nature and charged SCAmpPs are almost exclusively positive (Figure 2, Additional file 2), consistent with disruption of membrane permeability. The presence of the expected SCAmpPs-4 cyclic peptide in the phloem was confirmed by ESI-MS. In addition, these analyses suggested a non-covalent SCAmpPs-4-potassium complex. Relative binding efficiencies in non-covalent complexes, including polypeptide metal complexes, are known to be reflected in ESI-MS analyses [25]. The data is therefore consistent with strong binding of potassium by SCampPs-4 peptide in phloem, and opens the possibility that this peptide acts as a carriertype ionophore capable of transporting $\mathrm{K}+$ cations across a membrane [32].

The majority of the SCAmpPs loci identified on the $C$. sinensis and $C$. clementina genome assemblies are not annotated. This is not unexpected given the small size of the precursor product, the interruption of this small domain by an intron, and the rapid divergence of genes for ribosomally synthesized peptide products [33].

While processing of the precursor to the cyclic product is unusual, the SCAmpPs-3 and SCAmpPs-4 genes also display an extraordinary evolutionary profile. The hypervariability of the 15-24 bp peptide domain (Figures 3 and 4) stands in contrast to the unexpected conservation of the $2.5 \mathrm{~kb}$ upstream from the peptide region (Figure 5). While the SCAmpPs-3 and SCAmpPs-4 genes encode radically different final cyclic products, these genes share $>99.9 \%$ identity for the $2.5 \mathrm{~kb} 5^{\prime}$ to the peptide. This hyperconservation may in fact be related to the unusual 

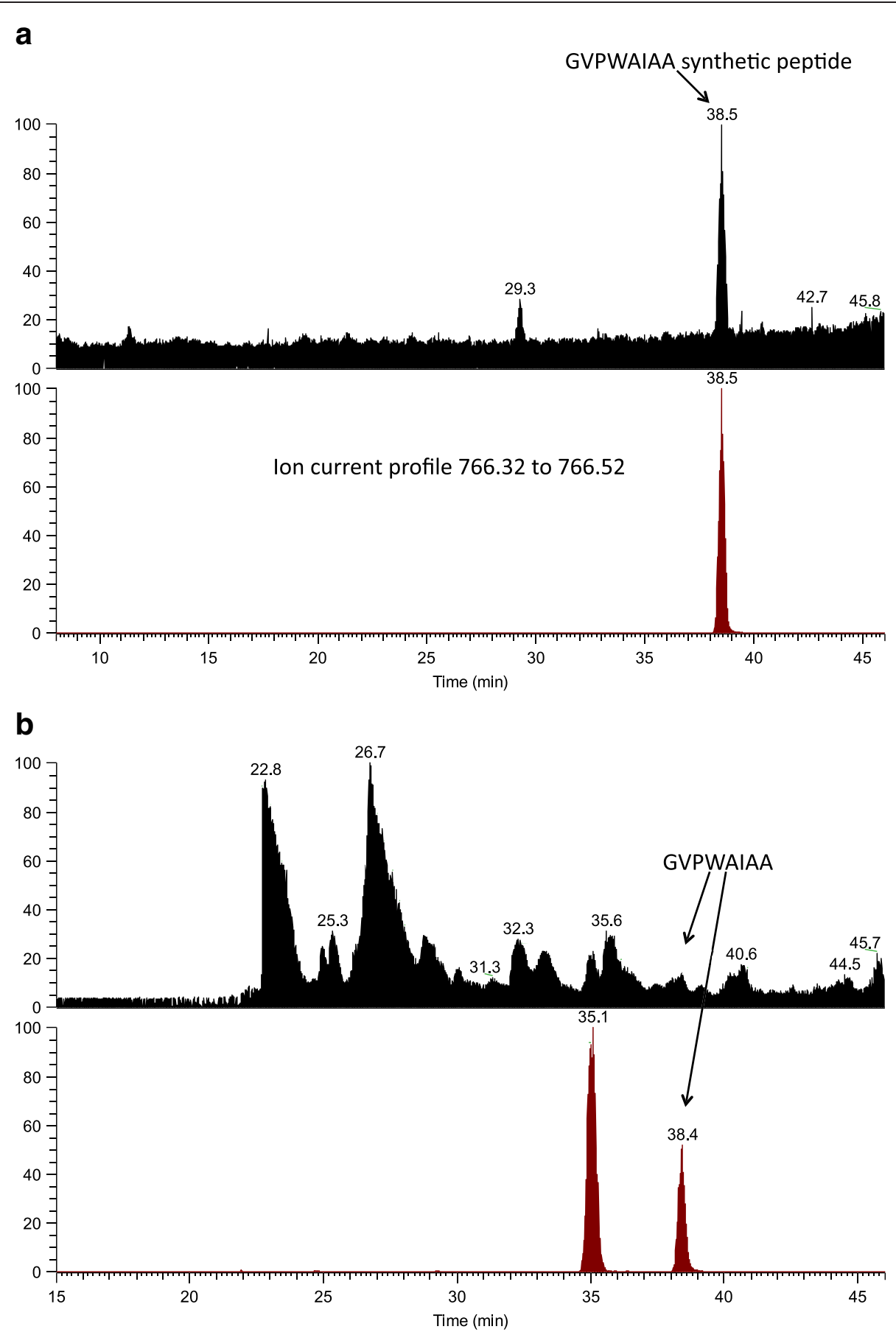

Figure 7 LC-MS of SCAmpPs cyclic peptides. (a). HPLC-MS chromatogram of synthetic peptide GVPWAIAA. Upper trace is total ion current. Lower traced is the extracted ion current profile for the molecular ion $\left(\mathrm{MH}^{+}\right)$of cyclic GVPWAIAA. (b). HPLC-MS chromatogram of the fractionated phloem extract. Upper trace is total ion current (TIC). Lower trace is ion current profile for $766.32-766.52 \mathrm{~m} / \mathrm{z}$ consistent with the molecular ion $\left(\mathrm{MH}^{+}\right)$of GVPWAIAA. Peak at 38.4 minutes is consistent with retention time of synthetic cyclic peptide GVPWAIAA.

transcriptional profiles of the SCAmpPs-3 and SCAmpPs-4 genes. They are equally represented in the phloem cDNA library, each contributing $>1 \%$ of the total transcripts. The maintenance of this level of transcription may preclude the expected accumulation of minor sequence changes (SNPs).

\section{Conclusion}

We report here the description of the SCAmpPs gene family from citrus. The SCAmpPs predicted cyclic products have a number of properties that are consistent with designation as potential ribosomally synthesized 
antimicrobial peptides, including peptide structure, amino acid composition and potential cation binding properties. In addition, the expression patterns, evolutionary profile and chromosomal distribution of the genes encoding these peptides are consistent with this assignment in allowing evolutionary flexibility to respond to biotic challenges.

\section{Methods}

\section{Identification of SCAmpPs genes}

SCAmpPs genes were identified on the Citrus clementina (assembly v1.0) [12] and Citrus sinensis (assembly v1.0) [11] assemblies by Pustell matrix analysis [34] of individual down-loaded chromosomes (MacVector11.1) using the SCAmpPs precursor peptide consensus sequence (Figure 2). Individual SCAmpPs coding domains and flanking regions were then used in BLAST searches of the NCBI citrus EST database to identify related ESTs.

\section{PCR amplification of SCAmpPs-3 and SCAmpPs-4 genes} SCAmpPs genes were amplified from Citrus genomic DNA using synthetic oligonucleotide primers. SCAmpPs-3 and SCAmpPs-4 were amplified from C. clementina genomic DNA using oligos (For-WRB 2236 GTTAC AGTTATGAACCCCTAACATTACTC and Rev-WRB 2239 ATAGAACATTTAAGATCGATGCTTAGC) based on the C. clementina genomic scaffolds. SCAmpPs-3 was also amplified from Carrizo citrange (Citrus sinensis 'Washington' sweet orange $\mathrm{X}$ Poncirus trifoliata) DNA using oligos (For-WRB 2228 CAGTTATGAACCCCTAACATTACT CATCC and Rev-WRB 2230 CTTTAGCACAAAGAGA TCTCGATTCTC) based on the C. sinensis (For) and Citrus consensus (Rev) scaffold regions, respectively. PCR amplification was performed for 30 cycles using Phusion $2 x$ High Fidelity Master Mix with HF buffer at manufacturer's suggested annealing temperatures and conditions (New England Biolabs, Inc.-NEB). Amplified products were analyzed by $1 \%$ agarose gel electrophoresis (AGE), and PCR products were cloned directly into $\mathrm{PCR}$ Blunt II TOPO vector (Invitrogen ${ }^{\mathrm{nw}} /$ Life Technologies) using the quick ligase protocol (NEB). Subsequent clones were identified via EcoRI restriction endonuclease digestion and AGE. SCAmpPs sequences were confirmed using fluorescent dideoxy sequencing of both strands employing overlapping synthetic oligonucleotide primers. Sequences were aligned, edited and compared (MacVector11.1). Complete sequences of the genomic PCR products are available on the USDA Public Citrus Genome Database (http:// citrus.pw.usda.gov/).

\section{LC-MS-MS of the SCAmpPs-4 Synthetic Peptide and from} Citrus clementina phloem

The SCAmpPs-4 s synthetic cyclic peptide GVPWAIAA was acquired from AnaSpec (Fremont, CA). Phloem tissue was prepared from the bark of nursery-grown $C$. clementina trees and powered under liquid nitrogen. A SCAmpPs cyclic peptide-enriched fraction was prepared by fractionating the methanol-soluble constituents from the phloem powder with ethyl acetate and ethanol and resuspended in water [35].

The synthetic peptide was solubilized with $0.1 \%$ formic acid in water (Optima LC/MS grade, Fisher Scientific, Waltham, MA), to approximately $1 \mathrm{pM} / \mathrm{uL}$. A portion of each sample was transferred to respective autosampler vials and placed in the autosampler of an EASY-nLC II interfaced to an Orbitrap Elite mass spectrometer (Thermo Scientific, San Jose, California) with a PicoChip nanospray source (New Objectives, Woburn, MA). For each LC-MS run, a $2 \mathrm{uL}$ portion of sample was loaded by the autosampler onto an EASY-column trap ( $2 \mathrm{~cm}$, ID $100 \mu \mathrm{m}, 5 \mu \mathrm{m}$, $120 \AA$, ReproSil-Pur C18-AQ) and washed with 16uL solvent $\mathrm{A}$, then switched in-line with a $75 \mu \mathrm{m}$ ID column containing $10 \mathrm{~cm}$ of $3 \mu \mathrm{m}, 120 \AA$, ReproSil-Pur C18-AQ reverse phase packing (New Objectives, Woburn, MA). Samples were eluted into the Thermo Scientific Orbitrap Elite mass spectrometer with a binary gradient flow at a rate of $200 \mathrm{~nL} /$ minute. Solvent A was water, and Solvent B was acetonitrile, both solvents were Optima LC/MS grade containing $0.1 \%$ formic acid. The gradient was programed from $2 \%$ Solvent B to $16 \%$ Solvent B over 10 minutes, then to $36 \%$ solvent $\mathrm{B}$ at time 36 minutes, then to $80 \%$ solvent $\mathrm{B}$ at time 48 minutes, and held at $80 \%$ B for 8 minutes. Peptides were detected in the Orbitrap with the FT survey scan from 300 to $2000 \mathrm{~m} / \mathrm{z}$ at a resolution of 30,000. The 6 most intense peaks above a threshold of 30,000 counts were subject to higher-energy C-trap dissociation (HCD) with normalized collision energy set to 35 , default charge state set to 2, isolation width set to $2.0 \mathrm{~m} / \mathrm{z}$, and activation time set to $0.10 \mathrm{~ms}$. Product spectra were recorded at 30,000 resolution with the low mass set to $50 \mathrm{Da}$. Dynamic exclusion was enabled for duration of 10 seconds with a repeat count of 1 . Charge state screening allowed $+1,+2$, and +3 charge states to be selected for HCD fragmentation. Monoisotope precursor selection was enabled.

\section{Additional files}

Additional file 1: Positions of SCAmpPs genes and pseudogenes on the $C$. clementina genome assembly. Gene indicates representation ( $\geq 99 \%$ ) in the NCBI EST DATA base.

Additional file 2: Amino acid frequency of average citrus proteins compared to SCAmpPs peptides. (a) Average amino acid frequency calculated using C. sinensis Chr7 proteins. Amino acid frequency is consistent with Jordan et al. (b) Amino acid frequency of combined SCAmpPs cyclic peptides from Figure 2.

Additional file 3: Representation of SCAmpPs-2 (phloem-specific) and SCAmpPs-5 (abscission zone specific) in phloem and abscission zone EST libraries. Complete citrus phloem (LIBEST_017673) and abscission zone (LIBEST_019157) EST libraries were downloaded from $\mathrm{NCBI}$ and sequences placed into contiguous DNA files. The EST libraries 
were probed by DNA matrix analysis (35 bp window, $85 \%$ identity) using SCAmpPs-2 (NCBl accession DR909920) and SCAmpPs-5 (NCBI accession FC872925). SCAmpPs-2, together with related SCAmpPs-3 and SCAmpPs-4 transcripts make up 5.5\% of the transcripts in the phloem library. SCAmpPs-5, together with related SCAmpPs- 6 transcripts make up $4.9 \%$ of the transcripts in the abscission zone library.

Additional file 4: Alignment of related SCAmpPs ESTs. (a) Alignment of SCAmpPs ESTs similar to the gene at position 7.120 Mb C. clementina Chr5, NCBI accession numbers indicated. (b) Alignment of SCAmpPs ESTs (NCBI accession numbers indicated). Coding domains, mismatches, position of the intronsindicated as in Figure 3.

Additional file 5: Alignment of variable domains from SCAmpPs-3 and -4 promoters 130 bp $5^{\prime}$ to the transcription start. Sequences of 4 SCAmpPs-3 and 3 SCAmpPs-4 gemomic PCR products aligned. Missmatch base pairs in red.

\section{Additional file 6: Structrue of SCAmpPs pseudogene at position}

2.109 Mb C. clementina Chr5. Exon, coding, cyclic peptide and variable regions are indicated as in Figure 7. The yellow boxes indicate positions of repetitive SINE and MITE elements.

Additional file 7: MS-MS of SCAmpPs-4 synthetic peptide and phloem extract. (a) MS-MS spectrum of $\mathrm{m} / \mathrm{z} 766.42$ collected during HPLC run of synthetic cyclic peptide GVPWAIAA. HCD collision energy 35 volts. (b) MS-MS spectrum of $\mathrm{m} / \mathrm{z} 766.42$ collected during HPLC run of methanol extract of phloem. HCD collision energy 35 volts.

\section{Additional file 8: Mass spectral analysis of SCAmpPs-4} chromatographic peaks from synthetic peptide and phloem extract. (a) Synthetic peptide MS-1 mass spectrum chromatographic peak at 38.4 minutes. Peak at 766.4225 is consistent with calculated molecular weight of protonated cyclic peptide. Peak at 788.4038 is consistent with the calculated molecular weight of the sodium adduct of the cyclic peptide. Charge state (Z) and mass measurement error (given in parts per million) are indicated. (b) Methanol extract of phloem MS-1 mass spectrum chromatographic peak at 38.4 minutes. Peak at 766.4217 is consistent with calculated molecular weight of protonated cyclic peptide. Peak at 804.3769 is consistent with the calculated molecular weight of the potassium adduct of the cyclic peptide. Charge state (Z) and mass measurement error (given in parts per million) are indicated.

\section{Abbreviations}

HLB: Huanglongbing; ESI-MS: Electrospray lonization-Mass Spectrometry; HCD: Higher-energy C-trap dissociation; LC-MS: Liquid Chromatography- Mass Spectrometry; LC-MS-MS: Liquid Chromatography-Tandem Mass Spectrometry; NB-LRRP: Nucleotide Binding-Leucine Rich Repeat Protein; SCAmpPs: Small Cyclic Amphipathic Peptides.

\section{Competing interests}

The authors declare that they have no competing interests.

\section{Authors' contributions}

WRB conducted bioinformatics analyses. KFM amplified and sequenced the SCAmpPs genomic DNA products. LAH and WHV devised and executed the LC-MS analysis. MGB carried out phloem transcriptional analyses. WRB and ES coordinated the project and drafted the manuscript. All authors read and approved the final manuscript.

\section{Acknowledgements}

This work supported by the Agricultural Research Service National Programs (CRIS Project Numbers 5325-21220-001-00D and 5325-43000-028-00D).

\section{Author details}

'USDA-ARS, Western Regional Research Center, Albany, CA, USA. ²USDA-ARS, U. S. Horticultural Research Laboratory, Fort Pierce, FL, USA.

Received: 8 October 2014 Accepted: 25 March 2015 Published online: 16 April 2015

\section{References}

1. Marmiroli N, Maestri E. Plant peptides in defense and signaling. Peptides. 2014:56:30-44.
2. Grill E, Loffler S, Winnacker EL, Zenk MH. Phytochelatins, the heavy-metal-binding peptides of plants, are synthesized from glutathione by a specific gamma-glutamylcysteine dipeptidyl transpeptidase (phytochelatin synthase). Proc Natl Acad Sci U S A. 1989;86(18):6838-42.

3. Arnison PG, Bibb MJ, Bierbaum G, Bowers AA, Bugni TS, Bulaj G, et al. Ribosomally synthesized and post-translationally modified peptide natural products: overview and recommendations for a universal nomenclature. Nat Prod Rep. 2013;30(1):108-60.

4. Lay FT, Anderson MA. Defensins-components of the innate immune system in plants. Curr Protein Pept Sci. 2005;6(1):85-101.

5. Stover E, Stange RR, McCollum TG, Jaynes J, Irey M, Mirkov E. Screening antimicrobial peptides in vitro for use in developing transgenic citrus resistant to Huanglongbing and citrus canker. J Am Soc Hortic Sci. 2013;138(2):142-8.

6. Mozoruk J, Hunnicutt LE, Cave RD, Hunter WB, Bausher MG. Profiling transcriptional changes in Citrus sinensis (L.) Osbeck challenged by herbivory from the xylem-feeding leafhopper Homalodisca coagulata (Say) by CDNA macroarray analysis. Plant Science. 2006;170(6):1068-80.

7. Mozoruk J, Hunnicutt LE, Hunter WB, Bausher MG. CsV03-3 is a member of a novel gene family from citrus that encodes a protein with DNA binding activity and whose expression is responsive to defense signals and abiotic stress. J Plant Physiol. 2008;165(5):531-43.

8. Matsumoto T, Tashiro N, Nishimura K, Takeya K. New cyclic peptides from Citrus aurantium. Heterocycles. 2002;57(3):477-82.

9. Morita H, Enomoto M, Hirasawa Y, lizuka T, Ogawa K, Kawahara N, et al. Cyclonatsudamine A, a new vasodilator cyclic peptide from Citrus natsudaidai. Bioorg Med Chem Lett. 2007:17(19):5410-3.

10. Condie JA, Nowak G, Reed DW, Balsevich JJ, Reaney MJ, Arnison PG, et al. The biosynthesis of Caryophyllaceae-like cyclic peptides in Saponaria vaccaria L. from DNA-encoded precursors. Plant J. 2011;67(4):682-90.

11. Xu Q, Chen LL, Ruan X, Chen D, Zhu A, Chen C, et al. The draft genome of sweet orange (Citrus sinensis). Nat Genet. 2013;45(1):59-66.

12. Wu GA, Prochnik S, Jenkins J, Salse J, Hellsten U, Murat F, et al. Sequencing of diverse mandarin, pummelo and orange genomes reveals complex history of admixture during citrus domestication. Nat Biotechnol. 2014;32(7):656-62

13. Barber CJ, Pujara PT, Reed DW, Chiwocha S, Zhang H, Covello PS. The two-step biosynthesis of cyclic peptides from linear precursors in a member of the plant family Caryophyllaceae involves cyclization by a serine protease-like enzyme. J Biol Chem. 2013;288(18):12500-10.

14. Jordan IK, Kondrashov FA, Adzhubei IA, Wolf YI, Koonin EV, Kondrashov AS, et al. A universal trend of amino acid gain and loss in protein evolution. Nature. 2005:433(7026):633-8.

15. Brogden KA. Antimicrobial peptides: pore formers or metabolic inhibitors in bacteria? Nat Rev Microbiol. 2005;3(3):238-50.

16. Geer LY, Marchler-Bauer A, Geer RC, Han L, He J, He S, et al. The NCBI BioSystems database. Nucleic Acids Res. 2010;38(Database issue):D492-6.

17. Martinez-Godoy MA, Mauri N, Juarez J, Marques MC, Santiago J, Forment J, et al. A genome-wide $20 \mathrm{~K}$ citrus microarray for gene expression analysis. BMC Genomics. 2008;9:318.

18. Pfeil BE, Crisp MD. The age and biogeography of Citrus and the orange subfamily (Rutaceae: Aurantioideae) in Australasia and New Caledonia. Am J Bot. 2008;95(12):1621-31.

19. Woodward SR, Cruz L, Olivera BM, Hillyard DR. Constant and hypervariable regions in conotoxin propeptides. EMBO J. 1990;9(4):1015-20.

20. Conticello SG, Gilad Y, Avidan N, Ben-Asher E, Levy Z, Fainzilber M. Mechanisms for evolving hypervariability: the case of conopeptides. Mol Biol Evol. 2001;18(2):120-31.

21. Dewan KK. Secondary structure formations of conotoxin genes: a possible role in mediating variability. Biochem Bioph Res Co. 2006;349(2):701-8.

22. Chang D, Duda Jr TF. Extensive and continuous duplication facilitates rapid evolution and diversification of gene families. Mol Biol Evol. 2012;29(8):2019-29.

23. Michelmore RW, Christopoulou M, Caldwell KS. Impacts of resistance gene genetics, function, and evolution on a durable future. Annu Rev Phytopathol. 2013:51:291-319.

24. Bittner-Eddy PD, Beynon JL. The Arabidopsis downy mildew resistance gene, RPP13-Nd, functions independently of NDR1 and EDS1 and does not require the accumulation of salicylic acid. Mol Plant Microbe Interact. 2001;14(3):416-21.

25. Loo JA. Studying noncovalent protein complexes by electrospray ionization mass spectrometry. Mass Spectrom Rev. 1997;16(1):1-23. 
26. Rose MC, Henkens RW. Stability of Sodium and Potassium Complexes of Valinomycin. Biochimica Et Biophysica Acta. 1974;372(2):426-35.

27. Duax WL, Hauptman $\mathrm{H}$, Weeks CM, Norton DA. Valinomycin crystal structure determination by direct methods. Science. 1972;176(4037):911-4.

28. Finking R, Marahiel MA. Biosynthesis of nonribosomal peptides1. Annu Rev Microbiol. 2004;58:453-88.

29. Craik DJ. Circling the enemy: cyclic proteins in plant defence. Trends Plant Sci. 2009:14(6):328-35.

30. Nicolas P, Vanhoye D, Amiche M. Molecular strategies in biological evolution of antimicrobial peptides. Peptides. 2003;24(11):1669-80.

31. Papagianni M. Ribosomally synthesized peptides with antimicrobial properties: biosynthesis, structure, function, and applications. Biotechnol Adv. 2003;21(6):465-99.

32. Shapiro HM. Cell membrane potential analysis. Methods Cell Biol. 1994:41:121-33.

33. Schmidt EW. The hidden diversity of ribosomal peptide natural products. BMC Biol. 2010;8:83.

34. Pustell J, Kafatos FC. A convenient and adaptable microcomputer environment for DNA and protein sequence manipulation and analysis. Nucleic Acids Res. 1986;14(1):479-88.

35. Covello PS, Datla RSS, Stone SL, Balsevich JJ, Reaney MJ, Arnison PG, et al. DNA Sequences encoding caryophyllaceae and caryophyllaceae-like cyclopeptide precursors and methods of use. US Patent Application. US 2012/0058905 A1. In. 2012): 1-10.

\section{Submit your next manuscript to BioMed Central and take full advantage of:}

- Convenient online submission

- Thorough peer review

- No space constraints or color figure charges

- Immediate publication on acceptance

- Inclusion in PubMed, CAS, Scopus and Google Scholar

- Research which is freely available for redistribution 